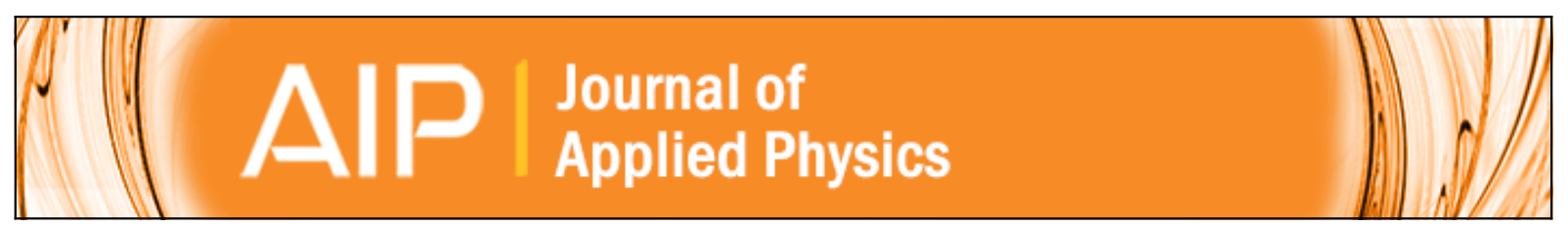

\title{
Thermally activated domain wall dynamics in a disordered magnetic nanostrip
}

Lasse Laurson, Claudio Serpico, Gianfranco Durin, and Stefano Zapperi

Citation: Journal of Applied Physics 109, $07 D 345$ (2011); doi: 10.1063/1.3565402

View online: http://dx.doi.org/10.1063/1.3565402

View Table of Contents: http://scitation.aip.org/content/aip/journal/jap/109/7?ver=pdfcov

Published by the AIP Publishing

\section{Articles you may be interested in}

Propagating and reflecting of spin wave in permalloy nanostrip with $360^{\circ}$ domain wall

J. Appl. Phys. 115, 013908 (2014); 10.1063/1.4861154

$360^{\circ}$ domain wall injection into magnetic thin films

Appl. Phys. Lett. 103, 222404 (2013); 10.1063/1.4828563

Phase diagram of magnetic domain walls in spin valve nano-stripes

Appl. Phys. Lett. 100, 172404 (2012); 10.1063/1.4704665

Influence of slanted nanostripe edges on the dynamics of magnetic domain walls

Appl. Phys. Lett. 97, 112508 (2010); 10.1063/1.3489359

Underlying mechanism of domain-wall motions in soft magnetic thin-film nanostripes beyond the velocitybreakdown regime

Appl. Phys. Lett. 93, 052503 (2008); 10.1063/1.2968138

\section{HDDEN}

\section{Instruments for Advanced Science}

Contact Hiden Analytical for further details: w www.HidenAnalytical.com E info@hiden.co.uk CLICK TO VIEW our product catalogue

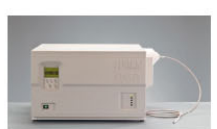

Gas Analysis

dynamic measurement of reaction gas streams catalysis and thermal analysis

molecular beam studies

dissolved species probes

fermentation, environmental and ecological studies

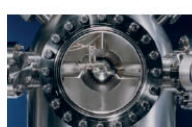

Surface Science

UHVTPD

SIMS end point detection in ion beam etch

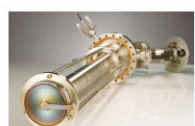

Plasma Diagnostics

' plasma source characterization etch and deposition process reaction kinetic studies , analysis of neutral and radical species

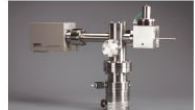

Vacuum Analysis partial pressure measurement and control of process gases reactive sputter process contro , vacuum diagnostics , acuum coating process monitorin 


\title{
Thermally activated domain wall dynamics in a disordered magnetic nanostrip
}

\author{
Lasse Laurson, ${ }^{1, a)}$ Claudio Serpico, ${ }^{2}$ Gianfranco Durin, ${ }^{3}$ and Stefano Zapperi ${ }^{4}$ \\ ${ }^{1}$ ISI Foundation, Viale S. Severo 65, 10133 Torino, Italy \\ 'ISI Foundation, Viale S. Severo 65, 10133 Torino, Italy and Universita di Napoli "Federico II" \\ Via Claudio 21,80125 Napoli, Italy \\ ${ }^{3}$ ISI Foundation, Viale S. Severo 65, 10133 Torino, Italy and INRIM, Strada delle Cacce 91, \\ 10135 Torino, Italy \\ ${ }^{4}$ ISI Foundation, Viale S. Severo 65, 10133 Torino, Italy and IENI-CNR, Via R. Cozzi 53, 20125, Milano, Italy
}

(Presented 16 November 2010; received 11 October 2010; accepted 6 January 2011; published online 12 April 2011)

\begin{abstract}
We consider the effect of disorder on the dynamics of a transverse domain wall in thin and narrow magnetic strips made of soft magnetic materials such as permalloy. Motivated by the micromagnetic description of such a domain wall, we construct a model based on two coupled flexible lines enclosing the domain wall transition region. The model captures both the finite width and the characteristic V-shape of the wall. Disorder is included via randomly distributed pinning centers interacting with the two lines, describing, e.g., thickness fluctuations of the strip. We study the thermally activated creep motion of the domain wall driven by an applied field in a disordered nanostrip, and demonstrate how such inherently stochastic motion leads to a broad distribution of the domain wall velocities, in agreement with experimental observations. (C) 2011 American Institute of Physics. [doi:10.1063/1.3565402]
\end{abstract}

\section{INTRODUCTION}

Many potential future memory and logic devices, including the racetrack memory ${ }^{1}$ and other applications, ${ }^{2-4}$ will rely on the use of the local magnetization of narrow and thin magnetic nanostrips or wires to store or process information. An important requirement for reliable operation of such devices is the ability to displace the magnetic domain walls (DWs) in a controlled fashion by applying field or current pulses along the strip/wire. A challenge is presented by various forms of disorder, such as roughness of the edges and surfaces of the sample, as well as various pointlike impurities in the bulk of the wire/strip. Such structural disorder, together with thermal fluctuations, will affect the DW dynamics especially for low driving forces. ${ }^{5-8}$ However, the majority of theoretical studies of DW dynamics in such systems has been considering "perfect" systems, free of any imperfections which in practice are always present in a realistic sample.

In this paper we study a simplified model of the transverse DW in thin and narrow nanostrips made of a soft magnetic material such as permalloy, ${ }^{9}$ taking into account "bulk" imperfections, such as thickness fluctuations of the strip, or various pointlike impurities present in the sample. The model is based on two flexible lines enclosing the transition region of the DW and provides a useful intermediate description between full micromagnetic modeling and models in which the DW is reduced into a $1 d$ magnetization profile. ${ }^{10}$ In particular, the model captures the finite width and the characteristic shape of the DW resembling a letter $\mathrm{V} .^{11}$

\footnotetext{
a) Author to whom correspondence should be addressed. Electronic mail: lasse.laurson@gmail.com.
}

Disorder describing, e.g., thickness fluctuations of the strip can also be included in a natural way as randomly distributed pinning centers interacting with the two lines. The paper is organized as follows: In the following section, the line-based model is presented, while in Section III some preliminary numerical results will be presented and compared qualitatively to experimental observations. We present our conclusions Section IV.

\section{THE LINE-BASED MODEL}

We consider here a strip of length $L$, width $W$, and thickness $D$, satifying $L \gg W \gg D$, made of a soft magnetic material such as permalloy. As the anisotropy energy in soft magnetic materials is dominated by shape anisotropy, the domains lie along the long axis of the strip. We focus here on the case where $W$ and $D$ are sufficiently small such that the stable DW structure is the so-called transverse wall. ${ }^{9}$

Due to the balance between exchange and magnetostatic interactions (the latter arising due to the magnetic charges within the DW with an asymmetric distribution, see Fig. 1), the equilibrium shape of the transverse DW resembles the letter V. ${ }^{9}$ Here we consider a simplified description of such a wall structure by assuming the wall to be composed of two coupled flexible lines enclosing the transition region of the DW (see the bottom panel of Fig. 1). Within this approximation the magnetic charges of the transverse wall are taken to be concentrated along these two lines (with the charge density depending on the local orientation of the line) and at the strip edges. These charges give rise to demagnetizing fields $\vec{H}_{d m}$ acting upon the segments of the two lines. Further ingredients of the model include line tension $\gamma_{w}$ of the lines, representing the energy cost due to deformations of the wall, as 


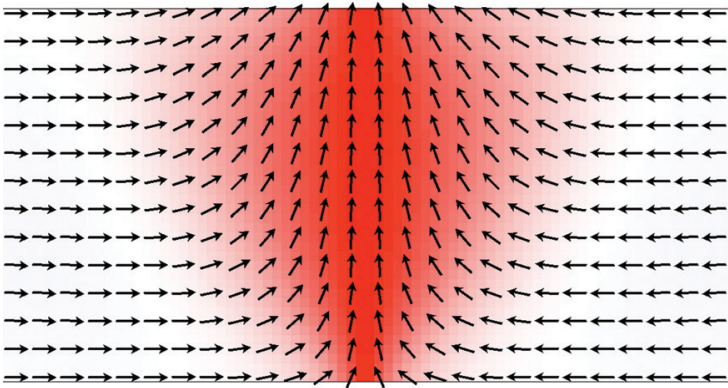

(a)

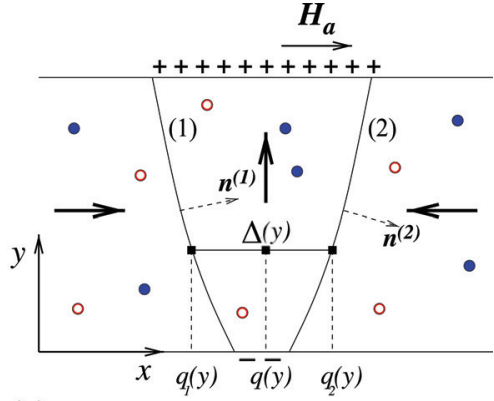

(b)
FIG. 1. (Color online) (a) The micromagnetic structure of the transverse wall. (b) A schematic representation of the line-based model, with the two coupled lines (1) and (2) enclosing the DW transition region. The model can be thought to consist of a set of " $1 d$ models," with widths $\Delta(y)=q_{2}(y)-q_{1}(y)$ and positions $q(y)$. The disorder is modeled by randomly distributed attractive and repulsive pinning sizes (open and filled circles, respectively). well as a repulsive interaction between the two lines, both originating from the exchange interactions.

As our model is a generalization of the so-called $1 d$ model for a $180^{\circ} \mathrm{DW}$ in a wire, we will first briefly review its main features. ${ }^{10,12}$ The magnetization profile $\vec{m}(x)$ along the wire ( $x$ axis) is described by the polar and azimuthal angles $\theta$ and $\phi$ via the ansatz

$$
\begin{gathered}
\theta(x, t)=2 \tan ^{-1} \exp \left[\frac{x-q(t)}{\Delta(t)}\right], \\
\phi(x, t)=\phi(t),
\end{gathered}
$$

where $q(t), \Delta(t)$, and $\phi(t)$ are the DW position, width, and (out-of-plane) magnetization angle, respectively. By expressing the various micromagnetic energy contributions (such as exchange, anisotropy, and applied field) in terms of these collective coordinates, one can derive from a Lagrangian formulation the equations of motion, or the Slonczewski equations, ${ }^{12}$ for $q(t), \phi(t)$, and $\Delta(t)$ :

$$
\begin{gathered}
\alpha \frac{\dot{q}}{\Delta}+\dot{\phi}=\gamma_{0} H_{a}, \\
\frac{\dot{q}}{\Delta}-\alpha \dot{\phi}=\gamma_{0} H_{K} \frac{\sin 2 \phi}{2}, \\
\dot{\Delta}=\frac{\gamma_{0}}{\alpha \mu_{0} M_{s} a}\left[\frac{A}{\Delta}-\left(K_{0}+K \sin ^{2} \phi\right) \Delta\right] .
\end{gathered}
$$

Here, $\alpha$ is the damping coefficient, $\gamma_{0}$ is the gyromagnetic ratio, $H_{a}$ is the applied field, $K_{0}$ and $K$ are (easy and hard axis, respectively) anisotropy constants, $H_{K}$ is the anisotropy field corresponding to $K$ (i.e. anisotropy perpendicular to the plane), $\mu_{0}$ is the vacuum permeability, $M_{s}$ is the saturation magnetization, $a=\pi^{2} / 12$, and $A$ is the exchange constant.

The line-based model is essentially a $2 d$ generalization of the $1 d$ model. The magnetization is described by the angles $\theta(x, y, t)$ and $\phi(t)$, with the former given by the $2 d$ ansatz

$$
\theta(x, y, t)=2 \tan ^{-1} \exp \left[\frac{x-q(y, t)}{\Delta(y, t)}\right]
$$

where the DW position $q(y, t)$ and width $\Delta(y, t)$ now depend upon the transverse coordinate $y$. Thus, the DW is modeled essentially as a set of $1 d$ profiles, with $y$-dependent positions and widths. The coordinates $q(y, t)$ are related to the coordinates $q_{1}(y, t)$ and $q_{2}(y, t)$ of the two lines enclosing the DW transition region via $q_{1}(y, t)=q(y, t)-\Delta(y, t) / 2$, and $q_{2}(y, t)$ $=q(y, t)+\Delta(y, t) / 2$.

By employing a similar procedure as in the case of the $1 d$ model, one obtains the equations of motion for the coordinates of the two lines,

$$
\begin{gathered}
\dot{q}_{1}=\frac{\Delta}{\alpha}\left(\gamma_{0} H_{a}-\dot{\phi}\right)-\frac{\gamma_{0}}{2 \alpha \mu_{0} M_{s} a}\left[\frac{A}{\Delta}-\Delta K \sin ^{2} \phi\right], \\
\dot{q}_{2}=\frac{\Delta}{\alpha}\left(\gamma_{0} H_{a}-\dot{\phi}\right)+\frac{\gamma_{0}}{2 \alpha \mu_{0} M_{s} a}\left[\frac{A}{\Delta}-\Delta K \sin ^{2} \phi\right], \\
\dot{\phi}=\frac{\gamma_{0}}{1+\alpha^{2}} H_{a}-\frac{\gamma_{0} \alpha}{2(1+\alpha)} H_{K} \sin 2 \phi .
\end{gathered}
$$

In addition, the exchange energy in the $y$ direction leads to an effective line tension which, together with the long-range magnetostatic interactions, couples the different " $1 d$ models." The latter are taken into account by approximating the distribution of magnetic charges in the domain wall by concentrating them on the two lines (as if they would represent two $90^{\circ}$ domain walls) and along the strip edges (see Fig. 1). These charges then give rise to magnetostatic fields acting on the segments of the two lines similarly to the applied field. Disorder is modeled via randomly distributed pinning centers which exert either an attractive or repulsive force on the DW representing, for instance, thickness fluctuations of the strip. In addition, we add a Gaussian white noise term $\eta(t, i)$ representing the effect of temperature, acting on the segments $i=1, \ldots, N$ of the two discretized lines, with correlations $\left\langle\eta(t, i) \eta\left(t^{\prime}, j\right)\right\rangle=D \delta\left(t-t^{\prime}\right) \delta(i-j)$. Here we restrict ourselves to consider the field-driven dynamics, however, extending the model to include the effect of a spin-polarized electric current is straightforward. Also, only fields well below the Walker field $H_{W}$ (Ref. 13) are considered, as the model does not capture the DW magnetization reversal taking place for $H_{a}>H_{w}$ in the strip geometry via nucleation and propagation of an antivortex. ${ }^{10}$

\section{NUMERICAL SIMULATIONS}

We study the thermally activated creep motion of the transverse DW by numerically integrating the equations of motion (7)-(9). We use material parameters of a soft magnetic material such as permalloy, $\alpha=0.01, A=1.5 \times 10^{-11} \mathrm{~J} / \mathrm{m}$, and $M_{s}=8.0 \times 10^{5} \mathrm{~A} / \mathrm{m}$. To study the effect of disorder and temperature on the DW dynamics, we first let the DW relax for $0.2 \mathrm{~ns}$ from an initial configuration close to the equilibrium $\mathrm{V}$-shape (with $\phi=0$ ), and then apply a field pulse of 


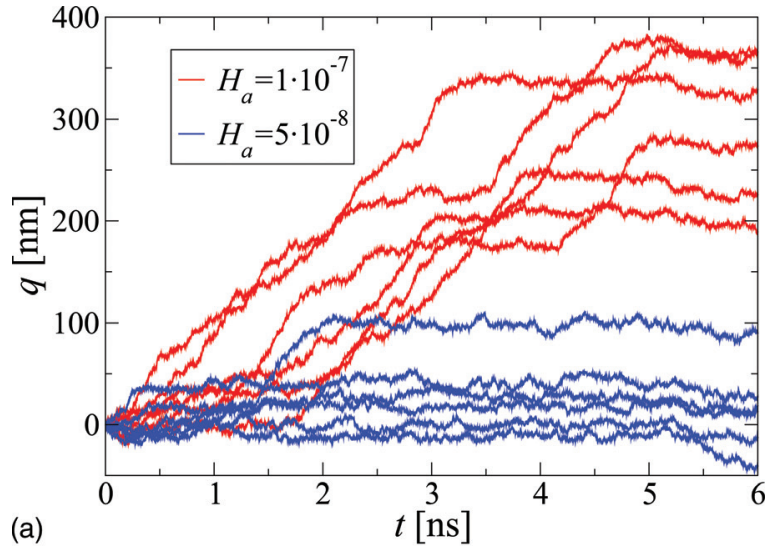

(a)

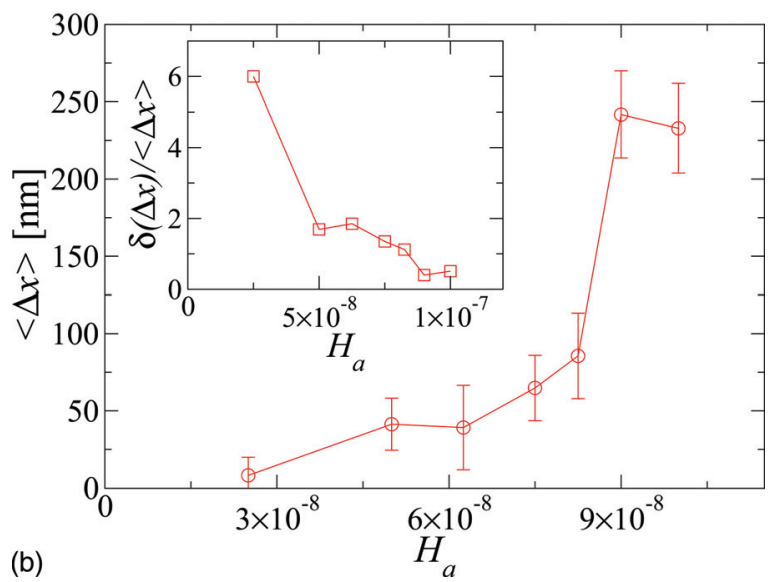

FIG. 2. (Color online) (a) Example trajectories of the DW during a field pulse as a function of time, with two different pulse amplitudes, $H_{a}$. (b) The average displacement of the DW during a field pulse as a function of the pulse strength. The error bars correspond to statistical error. The inset shows the relative fluctuations of the DW displacement.

$5.8 \mathrm{~ns}$ in duration, and monitor the induced displacement of the DW. The strength of the thermal fluctuations is fixed to be such that thermal activation plays a role within the time scales of the simulations, as shown in the top panel of Fig. 2 which shows the time evolution of the domain wall position $q$ for two different field pulse strengths $H_{a}$ (six example simulation runs each, with different realizations of the disorder). The DW dynamics in this low field regime is clearly stochastic, with a broad distribution of DW displacements: Sometimes the DW does not move at all during the applied field pulse, while some other runs lead to significant displacements of hundreds of nanometers. This is a typical feature of thermally activated subthreshold creep motion: The domain wall spends a significant fraction of the time pinned by the disorder, and occasionally jumps forward due to thermal activation. The average displacement $\langle\Delta x\rangle$ of the DW induced by the field pulse (shown in the bottom panel of Fig. 2) exhibits a nonlinear field dependence, typical of the creep motion of elastic manifolds. Such motion is inherently stochastic, and naturally leads to a broad distribution of the DW displacements/velocities, as evidenced by the inset of the bottom panel of Fig. 2: As the field pulse strength is decreased, the relative fluctuations of the DW displacement increase. Similar stochastic and broadly distributed DW velocities have also been observed in experiments. ${ }^{14}$

\section{CONCLUSIONS}

Our model serves as a useful intermediate description between full micromagnetic simulations and the $1 d$ models, capturing, for instance, the realistic V-shape of the transverse wall. The present version of the model also has the advantage that realistic physical units and parameters can be used in a transparent way. ${ }^{11}$ Also, it is particularly suitable for studies of the effect of disorder on the DW dynamics.

Numerical simulations of the model in the low field regime reveal stochastic creep motion of the DW, leading to a broad distribution of DW displacements and velocities. Such stochastic dynamics is also in qualitative agreement with some recent experimental observations. ${ }^{14}$ Future challenges include understanding in more detail the effect of various sources of disorder and thermal fluctuations on the DW dynamics in various regimes, including the low field/current creep regime studied here, and the high driving force regime above the Walker breakdown. Of particular importance for practical applications also is the effect of thermal fluctuations in the case of zero applied fields and currents, which should limit the lifetime of the "magnetic bits" stored in nanoscale wires and strips.

\section{ACKNOWLEDGMENTS}

L.L. wishes to thank the Academy of Finland for financial support.

${ }^{1}$ S. S. P. Parkin, M. Hayashi, and L. Thomas, Science 320, 190 (2008).

${ }^{2}$ G. S. D. Beach, M. Tsoi, and J. L. Erskine, J. Magn. Magn. Mater. 320, 1272 (2008).

${ }^{3}$ G. S. D. Beach, C. Nistor, C. Knutson, M. Tsoi, and J. L. Erskine, Nature Mater. 4, 741 (2005); R. Cowburn and D. Petit, Nature Mater. 4, 721 (2005).

${ }^{4}$ D. A. Allwood, G. Xiong, C. C. Faulkner, D. Atkinson, D. Petit, and R. P. Cowburn, Science 309, 1688 (2005).

${ }^{5}$ E. Martinez, L. Lopez-Diaz, L. Torres, C. Tristan, and O. Alejos, Phys. Rev B 75, 174409 (2007).

${ }^{6}$ Y. Nakatani, A. Thiaville, and J. Miltat, Nature Mater. 2, 521 (2003).

${ }^{7}$ M. T. Bryan, D. Atkinson, and R. P. Cowburn, J. Phys: Conf. Ser. 17, 40 (2005).

${ }^{8}$ E. Martinez, L. Lopez-Diaz, O. Alejos, L. Torres, and C. Tristan, Phys. Rev. Lett. 98, 267202 (2007).

${ }^{9}$ Y. Nakatani, A. Thiaville, and J. Miltat, J. Magn. Magn. Mater. 290-291, 750 (2005).

${ }^{10}$ A. Thiaville and Y. Nakatan, Spin Dynamics in Confined Magnetic Structures III, Topics in Applied Physics Vol. 101, edited by B. Hillebrands and A. Thiaville Springer-Verlag, Berlin, 2006), p. 161.

${ }^{11}$ L. Laurson, A. Mughal, G. Durin, and S. Zapperi, IEEE Trans. Magn. 46, 262 (2010).

${ }^{12}$ A. P. Malozemoff and J. C. Slonczewski, Magnetic Domain Walls in Bubble Materials (Academic, New York, 1979).

${ }^{13}$ N. L. Schryer and L. R. Walker, J. Appl. Phys. 45, 5406 (1974).

${ }^{14}$ G. Meier, M. Bolte, R. Eiselt, B. Krüger, D. H. Kim, and P. Fischer, Phys. Rev. Lett. 98, 187202 (2007). 